\title{
Monitoring circulating dipeptidyl peptidase 3 (DPP3) predicts improvement of organ failure and survival in sepsis: a prospective observational multinational study
}

\begin{abstract}
Alice Blet ${ }^{1,2,3^{*}} \mathbb{D}$, Benjamin Deniau ${ }^{1,2}$, Karine Santos $^{4}$, Dirk P. T. van Lier ${ }^{5,6}$, Feriel Azibani ${ }^{2}$, Xavier Wittebole ${ }^{7}$, Benjamin G. Chousterman 1,2, Etienne Gayat ${ }^{1,2}$, Oliver Hartmann ${ }^{8}$, Joachim Struck ${ }^{8}$, Andreas Bergmann ${ }^{4}$, Massimo Antonelli ${ }^{9}$, Albertus Beishuizen ${ }^{10}$, Jean-Michel Constantin ${ }^{11}$, Charles Damoisel ${ }^{1}$, Nicolas Deye ${ }^{2,12}$, Salvatore Di Somma ${ }^{13}$, Thierry Dugernier ${ }^{14}$, Bruno François ${ }^{15,16}$, Stephane Gaudry ${ }^{17}$, Vincent Huberlant ${ }^{18}$, Jean-Baptiste Lascarrou ${ }^{19}$, Gernot Marx ${ }^{20}$, Emmanuelle Mercier ${ }^{21}$, Haikel Oueslati ${ }^{1}$, Peter Pickkers ${ }^{5,6}$, Romain Sonneville ${ }^{22}$, Matthieu Legrand ${ }^{23}$, Pierre-François Laterre ${ }^{24}$ and Alexandre Mebazaa ${ }^{1,2}$ on behalf of AdrenOSS-1 Study Investigators
\end{abstract}

\begin{abstract}
Background: Dipeptidyl peptidase 3 (DPP3) is a cytosolic enzyme involved in the degradation of various cardiovascular and endorphin mediators. High levels of circulating DPP3 (CDPP3) indicate a high risk of organ dysfunction and mortality in cardiogenic shock patients.

Methods: The aim was to assess relationships between CDPP3 during the initial intensive care unit (ICU) stay and short-term outcome in the AdrenOSS-1, a prospective observational multinational study in twenty-four ICU centers in five countries. AdrenOSS-1 included 585 patients admitted to the ICU with severe sepsis or septic shock. The primary outcome was 28-day mortality. Secondary outcomes included organ failure as defined by the Sequential Organ Failure Assessment (SOFA) score, organ support with focus on vasopressor/inotropic use and need for renal replacement therapy. CDPP3 levels were measured upon admission and $24 \mathrm{~h}$ later.

Results: Median [IQR] cDPP3 concentration upon admission was 26.5 [16.2-40.4] ng/mL. Initial SOFA score was 7 [5-10], and 28-day mortality was 22\%. We found marked associations between CDPP3 upon ICU admission and 28-day mortality (unadjusted standardized HR 1.8 [Cl 1.6-2.1]; adjusted HR 1.5 [Cl 1.3-1.8]) and between CDPP3 levels and change in renal and liver SOFA score ( $p=0.0077$ and 0.0009 , respectively). The higher the initial cDPP3 was, the greater the need for organ support and vasopressors upon admission; the longer the need for vasopressor(s), mechanical ventilation or RRT and the higher the need for fluid load (all $p<0.005$ ). In patients with cDPP3 $>40.4 \mathrm{ng} /$ $\mathrm{mL}$ upon admission, a decrease in CDPP3 below $40.4 \mathrm{ng} / \mathrm{mL}$ after $24 \mathrm{~h}$ was associated with an improvement of organ
\end{abstract}

\footnotetext{
*Correspondence: alice.blet@aphp.fr

1 Department of Anesthesiology, Critical Care and Burn Center,

Lariboisière - Saint-Louis Hospitals, DMU Parabol, AP-HP Nord, University

of Paris, Paris, France

Full list of author information is available at the end of the article
}

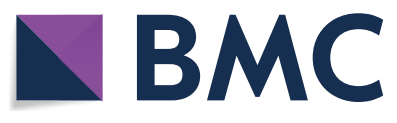

(c) The Author(s) 2021. Open Access This article is licensed under a Creative Commons Attribution 4.0 International License, which permits use, sharing, adaptation, distribution and reproduction in any medium or format, as long as you give appropriate credit to the original author(s) and the source, provide a link to the Creative Commons licence, and indicate if changes were made. The images or other third party material in this article are included in the article's Creative Commons licence, unless indicated otherwise in a credit line to the material. If material is not included in the article's Creative Commons licence and your intended use is not permitted by statutory regulation or exceeds the permitted use, you will need to obtain permission directly from the copyright holder. To view a copy of this licence, visit http://creativecommons.org/licenses/by/4.0/. The Creative Commons Public Domain Dedication waiver (http://creativeco mmons.org/publicdomain/zero/1.0/) applies to the data made available in this article, unless otherwise stated in a credit line to the data. 
function at $48 \mathrm{~h}$ and better 28-day outcome. By contrast, persistently elevated cDPP3 at $24 \mathrm{~h}$ was associated with worsening organ function and high 28-day mortality.

Conclusions: Admission levels and rapid changes in CDPP3 predict outcome during sepsis.

Trial Registration ClinicalTrials.gov, NCT02393781. Registered on March 19, 2015.

Keywords: DPP3, Biomarker, Outcome, Sepsis, Septic shock, Organ dysfunction

\section{Introduction}

Circulating dipeptidyl peptidase 3 (cDPP3) is an ubiquitous, intracellular, peptidase involved in the degradation of various cardiovascular and endorphin mediators $[1,2]$. When cell injury occurs, cDPP3 is released into the circulation. Prognostic properties of cDPP3 have already been demonstrated in critically ill patients suffering from cardiogenic shock and burn [3-5]. Apart from its function as a predictive biomarker of clinical outcome, we further showed, in pre-clinical studies, that cDPP3 exerts negative inotropic effects, working as a cardiac depressant factor [6]. These deleterious effects were reversed by Procizumab, a monoclonal anti-DPP3 neutralizing antibody [3]. This implies that cDPP3 may not only be a prognostic marker, but may also exert deleterious cardiovascular effects, representing a therapeutic target.

In a pilot study of septic patients, elevated CDPP3 levels upon ICU admission were observed in septic shock patients and in non-survivors [7]. In the present ancillary study from the Adrenomedullin and Outcome in Sepsis and Septic Shock 1 (AdrenOSS-1) study [8], we further investigated whether initial and repeated cDPP3 measurements during the early phase of sepsis may predict evolution of organ function and 28-day survival.

\section{Materials and methods \\ Study design}

AdrenOSS-1 was a prospective, observational study performed in 24 centers from five European countries (France, Belgium, The Netherlands, Italy and Germany) [8]. Patients $(n=585)$ were recruited from June 2015 to May 2016. The study protocol was approved by the local ethics committees and was conducted in accordance with Directive 2001/20/EC, as well as Good Clinical Practices (I.C.H. version 4 of May 1, 1996 and Decision of November 24, 2006) and the declaration of Helsinki. Criteria were previously described [8]. Briefly, main inclusion criteria was patients admitted to the ICU for severe sepsis or septic shock based on the former sepsis definition [9]. The primary endpoint was 28-day mortality. Secondary endpoints included organ failure (as defined by the SOFA score) and organ support, vasopressor/inotrope use, fluid balance and use of renal replacement therapy (RRT) and mechanical ventilation [10]. The use of organ support was left to the discretion of the clinician in charge of the patient in accordance with current practice. Acute kidney injury (AKI) was defined by KDIGO classification. Etiologies of AKI were not reported.

\section{Collection of patient data}

Upon admission, demographics (age, sex), body mass index (BMI), origin of sepsis, presence of shock, type of ICU admission, organ dysfunction scores (Sequential Organ Failure Assessment, SOFA; Acute Physiologic Assessment and Chronic Health Evaluation II, APACHE II), pre-existing comorbidities (i.e. treated within the last year), past medical history, laboratory values, as well as organ support were recorded and research blood was collected for measurement of cDPP3 and other markers.

After patient enrolment, the following data were collected daily during the first week: SOFA score, antimicrobial therapies, fluid balance, ventilation status, Glasgow coma scale, central venous pressure, need for RRT, invasive procedures for sepsis control and vasopressor/ inotrope treatment. Moreover, discharge status and mortality were recorded up to day 28 after ICU admission.

\section{Sample collection}

Blood for the central laboratory was sampled within $24 \mathrm{~h}$ after ICU admission and $24 \mathrm{~h}$ after the first sample. Samples were subsequently processed and plasma stored at $-80^{\circ} \mathrm{C}$ before transfer to the central lab for blinded cDPP3 measurement organized by the study sponsor [7] (4TEEN4 Pharmaceuticals GmbH, Hennigsdorf, Germany).

\section{Statistical analyses}

Results are presented as number ( $n)$ and percentage (\%), or median and inter-quartile range (IQR), as appropriate. Group comparisons for continuous variables were performed using the Kruskal-Wallis test. Categorical data were compared using Pearson's Chi-squared Test for Count Data. Biomarker data were log-transformed, if necessary.

Cox proportional-hazards regression was used to analyse the effect of risk factors on survival in uni- and multivariable analyses. The assumptions of proportional hazard were tested for all variables. For continuous variables, hazard ratios (HR) were standardized to describe the HR for a biomarker change of one IQR. 95\% 
confidence intervals (CI) for risk factors and significance levels for chi-square (Wald test) are given. The predictive value of each model was assessed by the model likelihood ratio chi-square statistic. The concordance index $(\mathrm{C}$ index) is given as an effect measure. It is equivalent to the concept of AUC adopted for binary outcome. For multivariable models, a bootstrap corrected version of the $\mathrm{C}$ index is given. To test for added predictive value, we used the likelihood ratio chi-square test for nested models to assess whether cDPP3 adds predictive value to a clinical model or a risk score. To assess the calibration accuracy of a multivariable Cox regression model, estimates of predicted survival probability will be plotted against observed survival in the data set. To this end, samples will be grouped into up to five groups according to their predicted survival, ensuring a minimum of 100 samples per group. Bootstrap corrected estimates are reported. Time-dependent Cox regression was used to evaluate the association of serial biomarker data and mortality. Survival curves plotted by the Kaplan-Meier method using quartiles of cDPP3 were used for illustrative purposes. Receiver-operating-characteristic (ROC) curves were constructed to illustrate the predictive performance for dichotomized endpoints. When dichotomizing the population based on CDPP3 was needed, the 3rd quartile was used $(40.4 \mathrm{ng} / \mathrm{mL})$, as the median $(26.5 \mathrm{ng} / \mathrm{mL})$ was within the normal range and therefore not a clinically meaningful cut point (upper normal range of DPP3 in plasma of healthy individuals is $41.2 \mathrm{ng} / \mathrm{mL}$ ).

A two-sided p-value of 0.05 was considered statistically significant. All analyses were performed using $\mathrm{R}$ version 3.4.3 (http://www.r-project.org, library rms, Hmisc) and Statistical Package for the Social Sciences (SPSS) version 22.0 (SPSS Inc., Chicago, Illinois, USA).

\section{Results}

Among the 585 patients included in AdrenOSS-1 study, 581 had a cDPP3 assessment. Admission demographics and details of clinical and biological parameters of the 581 septic patients are summarized in Table 1. Median [IQR] cDPP3 plasma levels upon admission in all Adren OSS-1 patients was $26.5 \mathrm{ng} / \mathrm{mL}$ [16.2-40.4].

We present the statistical results based on continuous data and used quartiles for categorization to visualize the results. High cDPP3 levels (defined by concentrations above the $3 r d$ quartile $(40.4 \mathrm{ng} / \mathrm{mL}$, which also represents the DPP3 upper normal range in healthy individuals) measured upon admission were associated with worse metabolic parameters, worse renal and cardiac functions, higher SOFA score, longer ICU stay and higher 28 and 90 day-mortality in comparison to low cDPP3 values (Table 1). Of note, patients with septic shock $(n=292)$ had a significantly higher cDPP3 concentration upon admission than patients with severe sepsis $(29.1 \mathrm{ng} / \mathrm{mL}$ [18.0-48.2] versus $23.2 \mathrm{ng} / \mathrm{mL}$ [15.2-35.1], $p=0.0001)$.

\section{cDPP3 levels upon ICU admission and mortality}

Over the 28-day follow-up period, 126 patients $(22 \%)$ died (33/289 (11\%) of patients diagnosed with severe sepsis and 93/292 (32\%) of patients diagnosed with septic shock). Admission characteristics of survivors versus non-survivors are shown in Additional file 8: Table 1. In univariate analysis, cDPP3 was associated with 28-day mortality (c index 0.692, standardized HR 1.8 [CI 1.62.1], $p<0.0001)$. For comparison, the $\mathrm{c}$ index for SOFA score was 0.729 (standardized HR 3.5 (2.6-4.6)) and for lactate 0.720 (standardized HR 1.8 (1.6-2.0)).

In a Cox proportional-hazard model adjusted for age, gender, comorbidities (cardiac or non-cardiac), diagnosis (severe sepsis, septic shock) and lactate, cDPP3 plasma levels upon admission remained independently associated with 28-day mortality (added chi-square 33.0, $p<0.0001$, c index increase from 0.756 to 0.776 , standardized HR 1.5 (1.3-1.8)). Additional file 1: Fig. 1 shows the calibration plot for the multivariable model. Overall, the model is well calibrated. A multivariate model further revealed that $\mathrm{CDPP} 3$ levels upon admission had incremental prognostic value on top of APACHE II or SOFA score (added chi-square 44.0 and 25.6, respectively, both $p<0.0001$ ), as well as on top of plasma lactate (added chi-square 43.6, $p<0.0001$ ) or procalcitonin (PCT, added chi-square $63.4, p<0.0001)$, when used as a continuous variable.

Kaplan-Meier analysis based on quartiles illustrates the association between cDPP3 plasma levels upon admission and 28-day mortality in all studied patients (Fig. 1). Notably, the survival rate for patients with cDPP3 levels above the 3rd quartile $(40.4 \mathrm{ng} / \mathrm{mL})$ sharply decreases within the first week (Fig. 1). Time-dependent AUC analysis of admission cDPP3 illustrates the superiority of cDPP3 compared to lactate and PCT for shortterm mortality prediction (Additional file 3: Fig. 3). This remains true in subgroups of severe sepsis and septic shock (Additional file 4: Fig. 4a, b). Additional file 2: Fig. 2 shows the ROC curve for association between cDPP3 and 28-day mortality.

\section{cDPP3 levels upon admission and organ dysfunction}

cDPP3 levels upon admission correlated with the initial SOFA score $(p<0.0001)$ (Additional file 5: Fig. 5). In addition, initial cDPP3 plasma levels were high in patients that had worsening of their total, renal and liver SOFA score in the first $48 \mathrm{~h}$ of ICU stay (Fig. 2).

Figure 3 further indicates that initial cDPP3 levels were elevated in 7-day survivors with need for organ support, high demand of vasopressors upon admission, and 
Table 1 Admission demographic patient characteristics

\begin{tabular}{|c|c|c|c|c|c|}
\hline Patient characteristics & All & Low CDPP3 & High cDPP3 & $p$ value* & $n$ \\
\hline Epidemiological data & $n=581$ & $n=436$ & $n=145$ & & \\
\hline cDPP3 at admission (ng/mL) & $26.5[16.2-40.4]$ & $19.8[14.5-28.9]$ & $67.7[48.7-120.7]$ & - & \\
\hline Age (year) & $66[55-75]$ & $66[56-76]$ & $65[53-75]$ & 0.2498 & \\
\hline Male (no. \%) & $363(62.5)$ & $277(63.5)$ & $86(59.3)$ & 0.4176 & \\
\hline Body mass index $\left(\mathrm{kg} / \mathrm{m}^{2}\right)$ & $25.7[22.9-30.2]$ & $25.5[22.7-30.1]$ & $26.1[23.4-31.1]$ & 0.1363 & \\
\hline Septic shock at admission (yes) & $292(50.3)$ & $201(46.1)$ & $91(62.8)$ & 0.0007 & \\
\hline Origin of sepsis & & & & 0.0727 & \\
\hline Lung & $218(37.5)$ & $151(34.6)$ & $67(46.2)$ & & \\
\hline Blood stream & $90(15.5)$ & $76(17.4)$ & $14(9.7)$ & & \\
\hline Urinary tract & $62(10.7)$ & $43(9.9)$ & $19(13.1)$ & & \\
\hline Catheter & $29(5.0)$ & $22(5)$ & $7(4.8)$ & & \\
\hline Peritonitis & $30(5.2)$ & $23(5.3)$ & $7(4.8)$ & & \\
\hline Endocarditis & $31(5.3)$ & $23(5.3)$ & $8(5.5)$ & & \\
\hline Other & $121(20.8)$ & $98(22.5)$ & $23(15.9)$ & & \\
\hline \multicolumn{6}{|l|}{ Medical history ${ }^{* *}$} \\
\hline Any cardiac comorbidity (yes) & $398(68.5)$ & $300(68.8)$ & $98(67.6)$ & 0.8642 & \\
\hline Chronic heart failure (yes) & $59(10.2)$ & $38(8.7)$ & $21(14.5)$ & 0.0652 & \\
\hline Hypertension (yes) & $292(50.3)$ & $220(50.5)$ & $72(49.7)$ & 1.0000 & \\
\hline Diabetes mellitus (yes) & $159(27.4)$ & $122(28)$ & $37(25.5)$ & 0.6286 & \\
\hline Any non-cardiac comorbidity (yes) & $413(71.1)$ & $308(70.6)$ & $105(72.4)$ & 0.7627 & \\
\hline Chronic renal disease (yes) & $76(13.1)$ & $57(13.1)$ & $19(13.1)$ & 1.0000 & \\
\hline Active/recent malignant tumors (yes) & $124(21.3)$ & $96(22)$ & $28(19.3)$ & 0.6090 & \\
\hline Smoking (active, yes) & $116(20)$ & $88(20.2)$ & $28(19.3)$ & 0.9238 & \\
\hline COPD (yes) & $89(15.3)$ & $70(16.1)$ & $19(13.1)$ & 0.5267 & \\
\hline Any chronic medication (yes) & $370(63.7)$ & $281(64.4)$ & $89(61.4)$ & 0.5712 & \\
\hline Immunosuppressive therapy (yes) & $46(7.9)$ & $32(7.3)$ & $14(9.7)$ & 0.4733 & \\
\hline \multicolumn{6}{|l|}{ Physiological values at admission } \\
\hline Mean blood pressure $(\mathrm{mmHg})$ & 75 [64-90] & 75 [65-90] & 76 [62-90] & 0.7372 & \\
\hline Heart rate (bpm) & 104 [89-119] & $101[88-117]$ & 108 [96-128] & 0.0005 & \\
\hline Fluid balance $(\mathrm{mL})$ & $1930[600-3556]$ & $1800[570-3270]$ & 2398 [805-4731] & 0.0059 & \\
\hline Urine output for $24 \mathrm{~h}(\mathrm{~mL})$ & 1000 [450-1900] & $1130[571-2000]$ & 600 [202-1495] & $<0.0001$ & \\
\hline $\mathrm{PaO}_{2} / \mathrm{FiO}_{2}$ & 228 [137-342] & $237[146-364]$ & 191 [111-300] & 0.0012 & \\
\hline \multicolumn{6}{|l|}{ Laboratory values at admission } \\
\hline Lactate $(\mathrm{mmol} / \mathrm{L})$ & $1.4[1.0-2.2]$ & $1.3[0.9-2]$ & $2.1[1.2-3.48]$ & $<0.0001$ & $n=560$ \\
\hline Arterial pH & $7.38[7.30-7.44]$ & $7.4[7.32-7.45]$ & $7.33[7.24-7.41]$ & $<0.0001$ & \\
\hline Bilirubin $(\mu \mathrm{mol} / \mathrm{L})$ & $11[6-19]$ & $10[6-18]$ & $12[7-22]$ & 0.1141 & \\
\hline Platelets $\left(10^{9} / \mathrm{L}\right)$ & $190[121-274]$ & $190[123-273.75]$ & 190 [112-269] & 0.7341 & \\
\hline Creatinine (mg/dL) & $1.35[0.86-2.24]$ & $1.26[0.81-2.05]$ & $1.71[1.04-2.9]$ & 0.0001 & \\
\hline BUN or urea $(\mathrm{mg} / \mathrm{dL})$ & $61.3[37.0-106.3]$ & $58.3[34.2-97]$ & $72[44-120.1]$ & 0.0012 & \\
\hline Hematocrit (\%) & $34[29-38]$ & $34[29-38]$ & $35[30-39]$ & 0.0395 & \\
\hline White blood count (per mm3) & $12,480[7200-18560]$ & $13,000[7560-18600]$ & 11,350 [6810-17040] & 0.0958 & \\
\hline Troponin T (ng/mL) & $42[18-152]$ & 38 [17-129] & $54[25-234]$ & 0.0786 & $n=152$ \\
\hline $\mathrm{PCT}(\mathrm{ng} / \mathrm{mL})$ & 11.4 [1.9-49.8] & $9.1[1.6-37.4]$ & $16.3[3.6-70.0]$ & 0.0194 & $n=330$ \\
\hline NT-proBNP (pg/mL) & 4382 [1525-11565] & 3339 [1294-7643] & 9589 [4863-20107] & 0.0003 & $n=117$ \\
\hline \multicolumn{6}{|l|}{ Organ support at admission } \\
\hline Mechanical ventilation: & & & & $<0.0001$ & \\
\hline Invasive & $217(37.3)$ & $134(30.7)$ & $83(57.2)$ & & \\
\hline Non-invasive & $131(22.5)$ & $98(22.5)$ & $33(22.8)$ & & \\
\hline None & $233(40.1)$ & $204(46.8)$ & $29(20)$ & & \\
\hline
\end{tabular}


Table 1 (continued)

\begin{tabular}{|c|c|c|c|c|c|}
\hline Patient characteristics & All & Low cDPP3 & High CDPP3 & $p$ value* & $n$ \\
\hline Renal replacement therapy & $49(8.4)$ & $19(4.4)$ & $30(20.7)$ & $<0.0001$ & \\
\hline Vasopressors/inotropes at admission & $347(59.7)$ & $246(56.4)$ & $101(69.7)$ & 0.0066 & \\
\hline \multicolumn{6}{|l|}{ Organ dysfunction scores } \\
\hline SOFA (points) & $7[5-10]$ & $7[4-9]$ & $9[5-12]$ & $<0.0001$ & $n=508$ \\
\hline APACHE II (points) & $15[11-20]$ & $15[11-19]$ & $18[13-23]$ & $<0.0001$ & \\
\hline \multicolumn{6}{|l|}{ Length of stay (days) } \\
\hline ICU & $5[2-10]$ & $5[2-8]$ & $6[2-15]$ & 0.0487 & \\
\hline \multicolumn{6}{|l|}{ Outcome } \\
\hline AKI within 7 days & $359(61.8)$ & $189(56.6)$ & $113(77.9)$ & $<0.0001$ & \\
\hline 28-day, deaths (\%) & $126(21.7)$ & $66(15.1)$ & $60(41.4)$ & $<0.0001$ & \\
\hline 90-day, deaths (\%) & $165(28.4)$ & $94(21.6)$ & $71(49)$ & $<0.0001$ & \\
\hline
\end{tabular}

Comparisons were performed between patients with cDPP3 above (high cDPP3) and below (low cDPP3) $40.4 \mathrm{ng} / \mathrm{mL}$ (3rd quartile) upon admission. APACHE Acute Physiology and Chronic Health Evaluation, BNP Brain-derived natriuretic peptide, BUN Blood urea nitrogen, CNS Central nervous system, COPD Chronic obstructive pulmonary disease, CDPP3 circulating Dipeptidyl peptidase 3, ICU Intensive care unit, NT-proBNP N-terminal brain natriuretic peptide, PaO2/ FiO2 Ratio of partial pressure of arterial oxygen to fraction of inspired oxygen, PCT Procalcitonin, SOFA Sequential Organ Failure Assessment Among the $n=121$ patients with origin of sepsis labelled as "other", source of infection were: bile duct infection $(n=12)$, CNS $(n=4)$, skin and soft tissue $(n=10)$, gynaecologic $(n=2)$, "unknown" ( $n=26)$, abdominal $(n=53)$, and other origins with less than 3 counts each $(n=14)$. * p Value from nonparametric Kruskal-Wallis or chi-square test, respectively. ${ }^{* *}$ Most common comorbidities reported individually

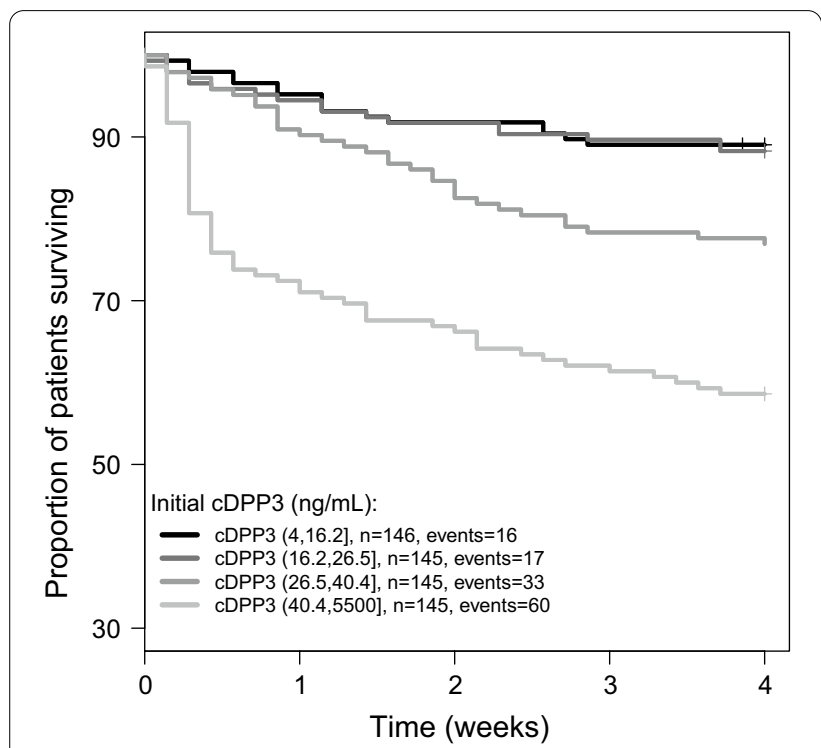

Fig. 1 Twenty-eight-day Kaplan-Meier survival curves for quartiles of CDPP3 at admission. Standardized HR is 1.8 [Cl 1.6-2.1], HR comparing patients with cDPP3 above and below $40.4 \mathrm{pg} / \mathrm{mL}$ (3rd quartile) is 3.4 [Cl 2.4-4.8]

prolonged vasopressor(s) need, as well as in patients that required mechanical ventilation, RRT and high fluid volume administration in the first week post admission. cDPP3 levels at admission were also significantly associated with need and duration of invasive mechanical ventilation $(p>0.0001)$ (Additional file 6: Fig. 6). Critically ill patients with high cDPP3 at admission were more likely to develop AKI within 7 days (Table 1). Further analyses showed greater cDPP3 levels in AKI versus nonAKI $(p=0.0001)$ (see Additional file 7: Fig. 7 for further details).

\section{Dynamic variation in CDPP3 levels and outcomes}

Combining admission and $24 \mathrm{~h}$ cDPP3 measurements via time-dependent Cox regression shows that the $24 \mathrm{~h}$ cDPP3 measurement provides significant added value on top of the admission value (added $\mathrm{Chi}^{2} 52.9, p<0.0001$ ). The added value of $24 \mathrm{~h} \mathrm{cDPP} 3$ remains an independent predictor of 28-day mortality after adjustment for SOFA score and lactate (added $\mathrm{Chi}^{2}$ 82.2, $p<0.0001$ ) or APACHE II score and lactate (added $\mathrm{Chi}^{2}$ 104.3, $p<0.0001$ ), with scores and lactate measured upon admission. For details see Fig. 4b. Figure 4a shows that changes in the concentrations of cDPP3 during the first $24 \mathrm{~h}$ were associated with different outcomes. Patients were divided into four groups based on admission and $24 \mathrm{~h}$ cDPP3 concentrations using the cut-off value of $40.4 \mathrm{ng} / \mathrm{mL}$ : remaining low (low-low, LL), admission high to low at $24 \mathrm{~h}(\mathrm{HL})$, admission low to high at $24 \mathrm{~h}(\mathrm{LH})$, and remaining high (high-high, $\mathrm{HH}$ ). Figure 4a shows that patients with dropping cDPP3 values from high levels upon admission to low levels after $24 \mathrm{~h}(\mathrm{HL})$ had reduced 28-day mortality risk compared to patients in whom cDPP3 levels remained high after $24 \mathrm{~h}(\mathrm{HH})(\mathrm{HR}$ 0.18 [0.08-0.41]). More importantly, the HL-patients had a similar risk of mortality compared to patients who remained low (LL) (HR 1.1 [0.52-2.4]). On the other hand, patients in whom cDPP3 became high after $24 \mathrm{~h}$ (LH) displayed an increased mortality risk compared to patients who started low and remained low (LL) (HR 2.2 

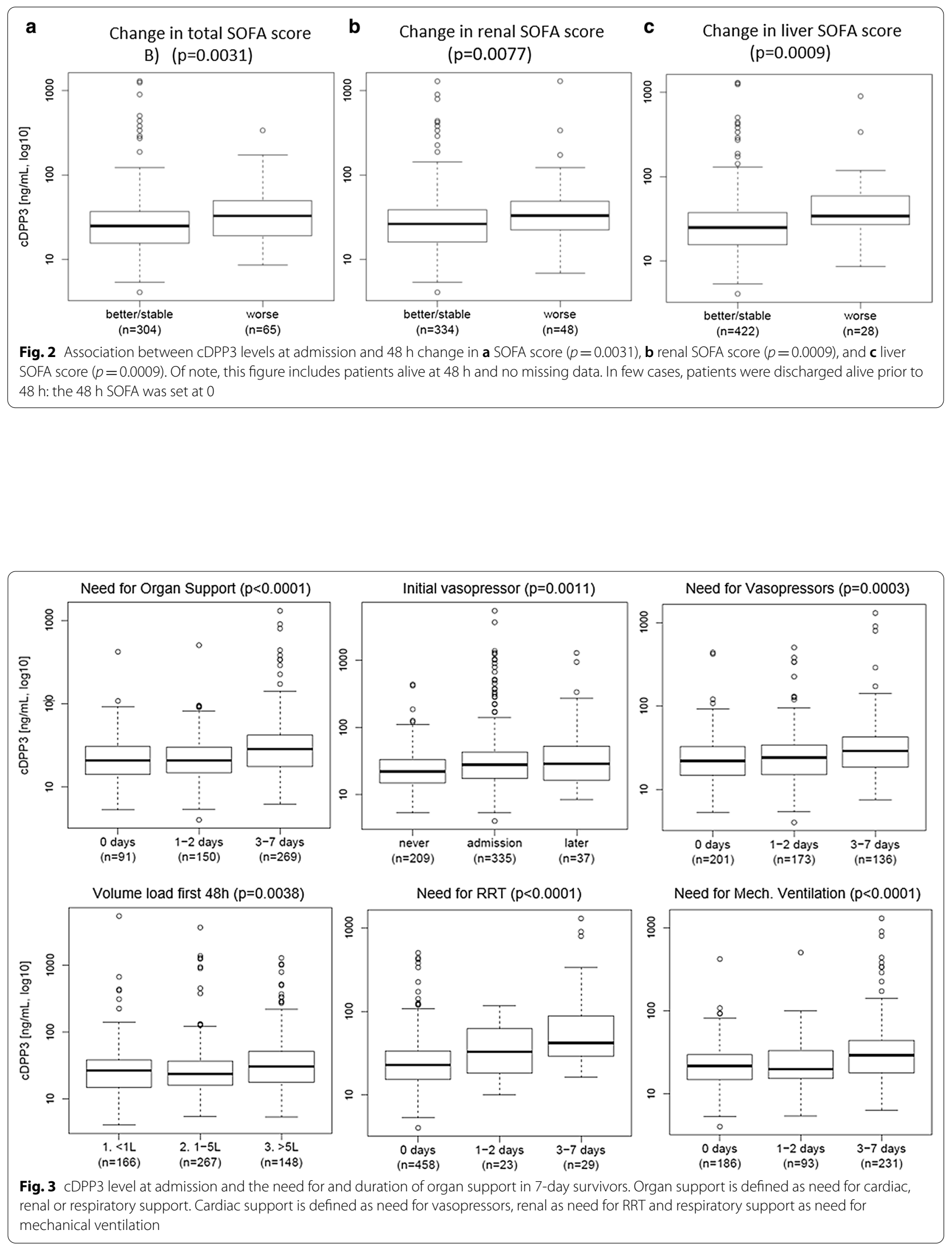


\section{(See figure on next page.)}

Fig. 4 Association between the changes of circulating DPP3 (cDPP3) levels over $24 \mathrm{~h}$ and mortality (A) Association between cDPP3 and 28-day mortality in time-dependent Cox regression, and worsening of SOFA score within $48 \mathrm{~h}$ in patients alive at $48 \mathrm{~h}(\mathbf{C}) \mathrm{HR}$ between high-high (HH) (levels of cDPP3 remained high) and high-low (HL) (levels of cDPP3 declining over $24 \mathrm{~h}$ ) 0.18 (95\% Cl 0.08-0.41). HR between low-low (LL) (levels of cDPP3 remaining low) and low-high (LH) (levels of CDPP3 increasing over $24 \mathrm{~h}$ ) 2.2 [1.0-4.8], $p=0.0015$ ). For patients discharged prior to $48 \mathrm{~h}$, the $48 \mathrm{~h}$ SOFA was set to 0

[CI 1.0-4.8]). Regarding organ function, regardless of the levels of cDPP3 upon admission, high concentrations of cDPP3 levels $24 \mathrm{~h}$ later ( $\mathrm{LH}$ and $\mathrm{HH}$ ) were associated with worsening of total SOFA score within $48 \mathrm{~h}$, as well as AKI (Fig. 4c and Additional file 7: Fig. 7c).

\section{Discussion}

This ancillary analysis of AdrenOSS-1 study, a prospective ICU multinational observational cohort study on biomarkers in sepsis, revealed that high cDPP3 levels upon admission had marked prognostic value and was associated with impaired short-term outcome. More importantly, early reduction of cDPP3 levels towards normal values was associated with improved organ function and 28-day survival, while persistent elevation of DPP3 predicted impaired outcome more accurately than current disease severity scores and e.g. lactate. Our study confirms previous work [7] but now in a larger cohort of ICU patients admitted with sepsis that cDPP3 upon admission is higher in septic shock compared to severely septic patients.

Our study further showed that patients admitted to the ICU with high cDPP3 levels, defined by values above the $3^{\text {rd }}$ quartile $(40 \mathrm{ng} / \mathrm{mL})$, had threefold greater risk of 28-day death. cDPP3 levels at admission were also elevated in patients with a worsening of total SOFA score, as well as worsening of renal and liver SOFA sub scores at $48 \mathrm{~h}$. In addition, high cDPP3 plasma levels were significantly associated with increased need for organ support, namely cardiovascular, mechanical ventilation and/ or renal replacement therapy in 7-day survivors. These results indicate that high cDPP3 plasma levels are associated with multiple organ injury and reflect patient severity. Our study also indicates that cDPP3 upon admission better predicts short-term mortality in sepsis than lactate or PCT. The marked prognostic value of CDPP3 for short-term mortality has been previously described in two cohorts of cardiogenic shock patients [3, 4]. In cardiogenic shock patients, levels of cDPP3 upon admission were higher than in septic shock patients and the threshold associated with higher short-term mortality was approx. $60 \mathrm{ng} / \mathrm{ml}$ compared to $40 \mathrm{ng} / \mathrm{ml}$ in septic patients [4]. Altogether, our study indicates that cDPP3 upon admission may be a promising prognostic factor in critically ill patients.
In addition to the prognostic value of cDPP3 upon admission, dynamics of cDPP3 during the initial phase were informative as well. We described marked association between low cDPP3 $(\leq 40.4 \mathrm{ng} / \mathrm{mL})$ at $24 \mathrm{~h}$ and low 28-day mortality, even in patients with initial high cDPP3 levels upon admission. Of interest, low cDPP3 at $24 \mathrm{~h}$ was also associated with improvement of organ function at day 7. Moreover, our study showed that prognostic properties of serial measurements of cDPP3 (admission and $24 \mathrm{~h}$ ) remained strong even when adjusted to ICU risk scores and lactate. Altogether, these findings indicate that CDPP3 measured once, or even better twice, in the first $24 \mathrm{~h}$ may markedly guide intensivists in the early management of septic patients.

Higher concentrations of cDPP3 were associated with more pronounced cardiovascular dysfunction, such as need for vasopressor therapy. Of interest, recent preclinical studies showed that the modulation of cDPP3 by specific antibodies may improve hemodynamic [3]. This implies that, apart from being a biomarker of cell damage, cDPP3 may exert functional properties and that treatment with an antibody may be beneficial in patients with elevated cDPP3 levels. Future studies should explore whether those novel therapies might improve organ function and survival in septic patients with high cDPP3.

Limitations of our study include its observational nature. The prognosis value of DPP3 as a biomarker will need to be confirmed in further studies. AdrenOSS-1 study was designed before the sepsis-3 definition. Therefore, the definition of severe sepsis and septic shock in AdrenOSS-1 was based on the "sepsis-2" definition [9]. It is, however, likely that cDPP3 plasma levels could have demonstrated similar prognostic properties in septic patients included with the sepsis-3 definition [11], especially as we show that $\mathrm{CDPP} 3$ has additional predictive properties over lactate. Furthermore, our data did not record parameters of myocardial function. We are, therefore, unable to link detrimental prognostic properties of cDPP3 to negative effects on heart contraction, as demonstrated in animals [3]. While we did show that higher cDDP3 concentrations are associated with greater need for hemodynamic support, further studies are clearly needed to fully clarify the impact of cDPP3 on cardiac contractility. In addition, our data did not record the indication for renal replacement therapy (RRT), neither the etiologies of AKI. Strengths of this study include its 


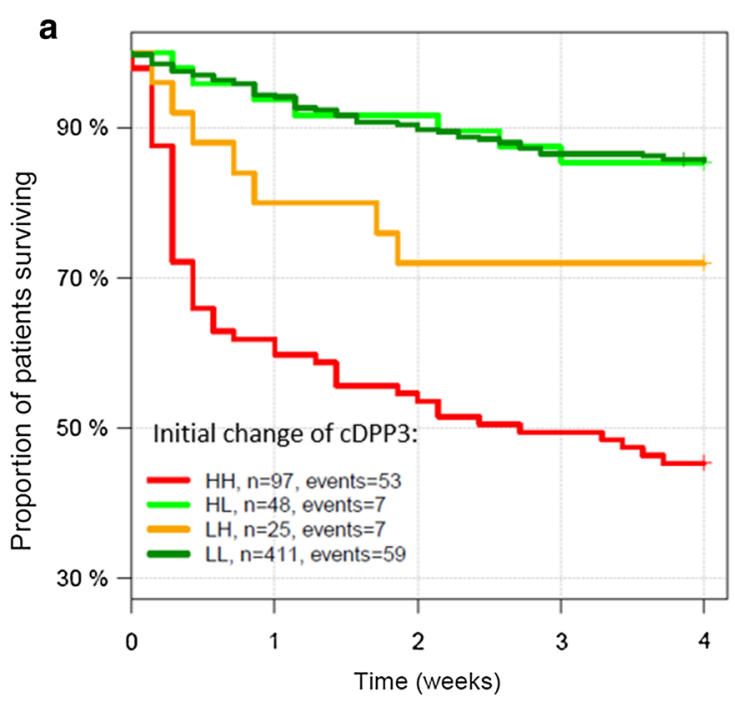

b

\begin{tabular}{|c|c|c|c|}
\hline Parameters & & Model $\mathrm{Chi}^{2}$ & $\begin{array}{c}\text { Added value } \\
\text { on top of cDPP3 } \\
\text { admission }\end{array}$ \\
\hline \multirow[b]{2}{*}{ cDPP3 } & cDPP3 admission & 68.2 & \\
\hline & $\begin{array}{c}\text { cDPP3 admission } \\
\text { \& cDPP3 24h }\end{array}$ & 121.1 & $<0.0001$ \\
\hline \multirow[b]{2}{*}{ SOFA score + lactate + cDPP3 } & cDPP3 admission & 74.4 & \\
\hline & $\begin{array}{c}\text { cDPP3 admission } \\
\text { \& cDPP3 } 24 \mathrm{~h}\end{array}$ & 156.6 & $<0.0001$ \\
\hline \multirow[b]{2}{*}{ APACHE II score + lactate + cDPP3 } & cDPP3 admission & 56.3 & \\
\hline & $\begin{array}{l}\text { cDPP3 admission } \\
\text { \& cDPP3 } 24 \mathrm{~h}\end{array}$ & 160.6 & $<0.0001$ \\
\hline
\end{tabular}

c

$50 \%$

$p=0.0015$

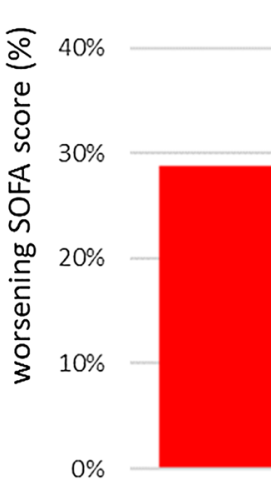

$\mathrm{HH}$

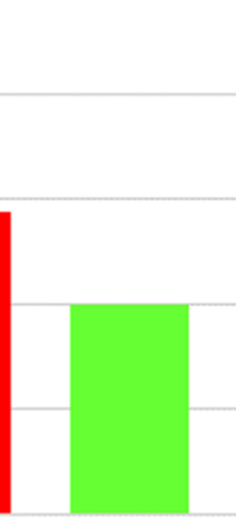

HL

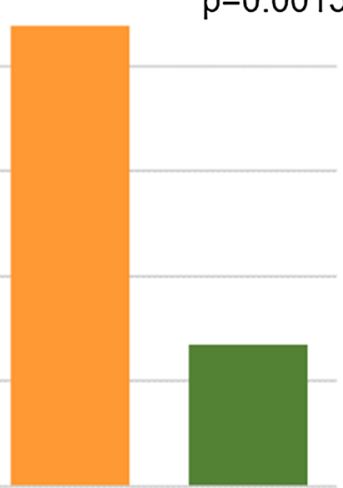

$\mathrm{LH}$

LL

initial change of cDPP3 
international conduct, indicating that the observed associations are generalizable and its considerable sample size, strengthening the point estimates of our findings.

\section{Conclusion}

In this ancillary analysis of AdrenOSS-1, we showed a strong association between high levels of cDPP3 in plasma upon ICU admission and clinical outcome, both short and mid-term. More importantly, normalization of cDPP3 levels in the first days after ICU admission was associated with marked improvement of total SOFA score and with lower 28-day mortality suggesting the need for cDDP3 monitoring during hospitalization. Combined with preclinical data demonstrating beneficial effects of DPP3 inhibition, these results warrant further investigation of the therapeutic potential to modulate the cDPP3 pathway in septic shock patients.

\section{Supplementary Information}

The online version contains supplementary material available at https://doi. org/10.1186/s13054-021-03471-2.

Additional file 1: Figure 1. Calibration plot designed for the multivariable model including age, gender, comorbidities (cardiac or non-cardiac), diagnosis (severe sepsis, septic shock), lactate and cDPP3 plasma levels upon admission for predicting 28-day mortality.

Additional file 2: Figure 2. ROC curve for association between CDPP3 and 28-day mortality. Based on the cut point $40.4 \mathrm{ng} / \mathrm{mL}$ (Q3) sensitivity was $47.6 \%$ and specificity $81.3 \%$.

Additional file 3: Figure 3. Time-dependent AUC plot for all-cause mortality for CDPP3, PCT and lactate, up to 28-days follow up. CDPP3 elicits the strongest prognostic properties in the first days.

Additional file 4:Figure $4 \mathrm{~A}$ and $\mathrm{B}$. Twenty-eight-day Kaplan-Meier survival curves for CDPP3 on admission, based on CDPP3 quartiles from the full population, for severe sepsis (A) and septic shock patients (B).

Additional file 5: Figure 5. Association of DPP3 and SOFA score at baseline $(p<0.0001)$.

Additional file 6: Figure 6. cDPP3 level at admission and the need for and duration of respiratory support, defined as invasive mechanical ventilation only $(p<0.0001)$.

Additional file 7: Figure 7A, B and C. (A) Box plot of CDPP3 level at admission upon AKI and non AKI patients. (B) ROC curve for association between CDPP3 and AKI. (C) Association between the changes of circulating DPP3 (CDPP3) levels over 48h and AKI.

Additional file 8: Table.

\section{Abbreviations}

APACHE II: Acute Physiologic Assessment and Chronic Health Evaluation II; AUC: Area Under Curve; BMI: Body Mass Index; CDPP3: Circulating Dipeptidyl Peptidase 3; Cl: 95\% Confidence intervals; DPP3: Dipeptidyl Peptidase 3; HR: Hazard Ratios; ICU: Intensive Care Unit; IQR: Inter-Quartile Range; RRT: Renal Replacement Therapy; SOFA: Sequential Organ Failure Assessment.

\section{Acknowledgements}

The authors are particularly grateful to Marie-Céline Fournier, who coordinated organizational aspects of the study. The authors also thank the Centre de Recherche Clinique (CRC) of Lariboisière University Hospital for support. Cliniques Universitaires Saint-Luc, Brussels, Belgium: Laterre. Clinique St Pierre, Ottignies, Belgium: Dugernier. Hôpital Jolimont, Haine-St-Paul, Belgium:
Huberlant. Klinik für Operative Intensivmedizin und Intermediate Care, Universitätsklinikum der RWTH, Aachen, Germany: Marx. Klinik für Anästhesiologie und Operative Intensivmedizin, Universitätsklinikum Köln, Köln, Germany: Hohn. HELIOS-Klinikum Erfurt, Erfurt, Germany: Meier-Hellmann. Klinikum Augsburg, Augsburg, Germany: Jaschinski. Klinik für Anästhesiologie und Intensivmedizin, Jena, Germany: Kortgen. CHU Dupuytren, Limoges, France: Francois. CHD les Oudairies, La Roche sur Yon, France: Lascarrou. CHU de Tours, Tours, France: Mercier. Centre hospitalier d'Angoulême, Angoulême, France: Desachy. CHU Angers, Angers, France: Lasocki. Hôpital Lariboisière, Paris, France (two centers) : Mebazaa. Hôpital Saint-Louis 1, Paris, France: Jacob. Hôpital Louis Mourier, Colombes, France: Gaudry. Hôpital Hautepierre, Strasbourg, France: Pottecher. CHU Estaing, Clermont Ferrand, France: Constantin. Hôpital Bichat Claude-Bernard, Paris, France: Sonneville. Sant'Andrea Hospital, Rome, Italy: Disomma. Policlinico Universitario A. Gemelli, Rome, Italy: Antonelli. Medisch Spectrum Twente, Enschede, The Netherlands: Beishuizen. UMC Radboudziekenhuis, Nijmegen, The Netherlands: Pickkers.

\section{Authors' contributions}

Involvement in conception and design of the study (PFL, AM, PP and GM), acquisition of the data ( $K S, X B, B C, E G, J S, A B e M A, A B, J M C, C D, N D, S d S, T D, B F$, $\mathrm{SG}, \mathrm{VH}, \mathrm{JBL}, \mathrm{EM}, \mathrm{HO}, \mathrm{RS}, \mathrm{ML}$ ), analysis and interpretation of the data (ABI, BD, FA, $\mathrm{OH}, \mathrm{AM})$, substantial involvement in the writing and/or revision of the article $(A B I, B D, K S, D V L, F A, O H, P P, A M)$; responsible for content of the manuscript including data and analysis (AM). All authors read and approved the final manuscript.

\section{Funding}

AdrenOSS-1 (ClinicalTrials.gov identifier NCT02393781) was funded by sphingotec GmbH, Neuendorfstraße 15a, 16761 Hennigsdorf, Germany. This project has received funding from the European Union's Horizon 2020 research and innovation program under grant agreement 666328.

\section{Availability of data and materials}

Data used for this study are available on request.

\section{Ethics approval and consent to participate}

The study protocol was approved by the local ethics committees and was conducted in accordance with Directive 2001/20/EC, as well as Good Clinical Practices (I.C.H. version 4 of May 1, 1996 and Decision of November 24, 2006) and the declaration of Helsinki.

\section{Consent for publication}

All authors have reviewed and approved the manuscript and are willing to attest to their qualifications as authors, disclose potential conflicts of interest, and release copyright should the manuscript be accepted for publication.

\section{Competing interests}

Dr. Mebazaa reports personal fees from Orion, Servier, Otsuka, Philips, Sanofi, Adrenomed, Epygon and Fire 1 and grants and personal fees from 4TEEN4, Abbott and Sphingotec. BD and ABI were invited to meetings in Hennigsdorf by 4TEEN4 Pharmaceuticals $\mathrm{GmbH}$. KS and ABe are employees of 4TEEN4 Pharmaceuticals $\mathrm{GmbH}$. BC served as a member of an advisory board for Roche Diagnostic. ML reports research support from sphingotec, lecture fees from Baxter and Fresenius, consulting fees from Novartis. Other authors declared no potential conflicts of interest with respect to the research authorship and/or publication of this article. 4TEEN4 Pharmaceuticals $\mathrm{GmbH}$ holds patent rights on the DPP3 biomarker and humanized antibody Procizumab. This does not alter our adherence to Critical Care Medicine policies on sharing data and materials.

\section{Author details \\ ${ }^{1}$ Department of Anesthesiology, Critical Care and Burn Center, Lariboisière - Saint-Louis Hospitals, DMU Parabol, AP-HP Nord, University of Paris, Paris, France. ${ }^{2}$ Inserm UMR-S 942, Cardiovascular Markers in Stress Conditions (MASCOT), University of Paris, 2 rue Ambroise Paré, 75010 Paris, France. ${ }^{3}$ University of Ottawa Heart Institute and University of Ottawa, Ottawa, ON, Canada. ${ }^{4}$ 4TEEN4 Pharmaceuticals GmbH, Hennigsdorf, Germany. ${ }^{5}$ Depart- ment of Intensive Care Medicine, Radboud University Medical Center, Geert Grooteplein Zuid 10, 6500 HB Nijmegen, The Netherlands. ${ }^{6}$ Radboud Center for Infectious Diseases, Radboud University Medical Center, Nijmegen, The} Netherlands. ${ }^{7}$ Department of Critical Care Medicine, St Luc University Hospital, 
Université Catholique de Louvain, Brussels, Belgium. ${ }^{8}$ SphingoTec GmbH, Hennigsdorf, Germany. ${ }^{9}$ Department of Anesthesiology and Intensive Care Medicine, Fondazione Policlinico Universitario A. Gemelli IRCCS, Rome, Italy.

${ }^{10}$ Department of Intensive Care, Medische Spectrum Twente, Enschede, The Netherlands. ${ }^{11}$ GRC 29, AP-HP, DMU DREAM, Department of Anaesthesiology and Critical Care, Pitié-Salpêtrière Hospital, Sorbonne University, Paris, France. ${ }^{12}$ Department of Medical and Toxicological Critical Care, Lariboisière Hospital, Federation of Toxicology APHP, Paris-Diderot University, Paris, France. ${ }^{13}$ Sant' Andrea Hospital, Rome, Italy. ${ }^{14}$ Clinique St Pierre, Ottignies, Belgium. ${ }^{15}$ ICU Department, CHU Dupuytren, Limoges, France. ${ }^{16}$ INSERM CIC 1435/UMR 1092, Limoges, France. ${ }^{17}$ Hôpital Louis Mourier, Colombes, France. ${ }^{18}$ Hôpital Jolimont, Haine-St-Paul, Belgium. ${ }^{19}$ Centre Hospitalier Universitaire de Nantes, Nantes, France. ${ }^{20}$ Klinik Für Operative Intensivmedizin Und Intermediate Care, Universitätsklinikum Der RWTH, Aachen, Germany. ${ }^{21} \mathrm{CHU}$ de Tours, Tours, France. ${ }^{22}$ Hopital Bichat Claude-Bernard, Paris, France. ${ }^{23}$ Department of Anesthesia and Perioperative Care, University of California San Francisco, San Francisco, USA. ${ }^{24}$ Department of Critical Care Medicine, Saint Luc University Hospital, Université Catholique de Louvain, Avenue Hippocrate 10, 1200 Brussels, Belgium.

Received: 22 October 2020 Accepted: 13 January 2021

Published online: 15 February 2021

\section{References}

1. Prajapati SC, Chauhan SS. Human dipeptidyl peptidase III mRNA variant I and II are expressed concurrently in multiple tumor derived cell lines and translated at comparable efficiency in vitro. MolBiol Rep. 2016;43:457-62.

2. Pang X, Shimizu A, Kurita S, Zankov DP, Takeuchi K, Yasuda-Yamahara M, et al. Novel therapeutic role for dipeptidyl peptidase III in the treatment of hypertension. Hypertens Dallas Tex. 1979;2016(68):630-41.

3. Deniau B, Rehfeld L, Santos K, Dienelt A, Azibani F, Sadoune M, et al. Circulating dipeptidyl peptidase 3 is a myocardial depressant factor: dipeptidyl peptidase 3 inhibition rapidly and sustainably improves haemodynamics. Eur J Heart Fail. 2019;
4. Takagi K, Blet A, Levy B, Deniau B, Azibani F, Feliot E, et al. Circulating dipeptidyl peptidase 3 and alteration in haemodynamics in cardiogenic shock: results from the OptimaCC trial. Eur J Heart Fail. 2019;

5. Dépret F, Amzallag J, Pollina A, Fayolle-Pivot L, Coutrot M, Chaussard $\mathrm{M}$, et al. Circulating dipeptidyl peptidase-3 at admission is associated with circulatory failure, acute kidney injury and death in severely ill burn patients. Crit Care LondEngl. 2020;24:168.

6. Deniau B, Blet A, Santos K, VaittinadaAyar P, Genest M, Kästorf M, et al. Inhibition of circulating dipeptidyl-peptidase 3 restores cardiac function in a sepsis-induced model in rats: a proof of concept study. PLOS ONE. 2020;15:e0238039.

7. Rehfeld L, Funk E, Jha S, Macheroux P, Melander O, Bergmann A. Novel methods for the quantification of dipeptidyl peptidase 3 (DPP3) concentration and activity in human blood samples. J Appl Lab Med. 2018. https ://doi.org/10.1373/jalm.2018.027995.

8. Mebazaa A, Geven C, Hollinger A, Wittebole X, Chousterman BG, Blet A, et al. Circulating adrenomedullin estimates survival and reversibility of organ failure in sepsis: the prospective observational multinational Adrenomedullin and Outcome in Sepsis and Septic Shock-1 (AdrenOSS-1) study. Crit Care LondEngl. 2018;22:354.

9. Levy MM, Fink MP, Marshall JC, Abraham E, Angus D, Cook D, et al. 2001 SCCM/ESICM/ACCP/ATS/SIS international sepsis definitions conference. Intensive Care Med. 2003;29:530-8.

10. Mebazaa A, Laterre PF, Russell JA, Bergmann A, Gattinoni L, Gayat E, et al. Designing phase 3 sepsis trials: application of learned experiences from critical care trials in acute heart failure. J Intensive Care. 2016;4:24.

11. Singer M, Deutschman CS, Seymour CW, Shankar-Hari M, Annane D, Bauer $\mathrm{M}$, et al. The third international consensus definitions for sepsis and septic shock (sepsis-3). JAMA. 2016;315:801-10.

\section{Publisher's Note}

Springer Nature remains neutral with regard to jurisdictional claims in published maps and institutional affiliations.
Ready to submit your research? Choose BMC and benefit from:

- fast, convenient online submission

- thorough peer review by experienced researchers in your field

- rapid publication on acceptance

- support for research data, including large and complex data types

- gold Open Access which fosters wider collaboration and increased citations

- maximum visibility for your research: over 100M website views per year

At BMC, research is always in progress.

Learn more biomedcentral.com/submissions 